\title{
Characterization of the reactivity and chlorinated products of carbazole during aqueous chlorination ${ }^{2 /}$
}

\author{
Xiong Xu ${ }^{a}$, Donghong Wang ${ }^{a,{ }^{*}}$, Chunmei Li ${ }^{a}$, Hongru Feng ${ }^{b}, Z^{2}$ jian Wang ${ }^{c}$ \\ a Key Laboratory of Drinking Water Science and Technology, Research Center for Eco-Environmental Sciences, Chinese Academy of Sciences, Beijing 100085, \\ China \\ b State Key Laboratory of Environmental Chemistry and Ecotoxicology, Research Center for Eco-Environmental Sciences, Chinese Academy of Science, Beijing \\ 100085, China \\ ' State Key Laboratory of Environmental Aquatic Chemistry, Research Center for Eco-Environmental Sciences, Chinese Academy of Sciences, Beijing 100085, \\ China
}

\section{A R T I C L E I N F O}

\section{Article history:}

Received 30 November 2016

Received in revised form

19 February 2017

Accepted 1 March 2017

Available online 9 March 2017

\section{Keywords:}

Carbazole

Chlorination

Fukui index

Comprehensive two-dimensional gas

chromatography

Reactivity

\begin{abstract}
A B S T R A C T
Carbazole in source water is a potential precursor for toxic chlorocarbazoles in drinking water when chlorine is used as a disinfection agent in drinking water treatment plants. However, the reactivity of carbazole and the specific structures and predominant analogues of chlorocarbazoles produced during aqueous chlorination remain unknown. In this study, the aqueous chlorination of carbazole was performed to characterize its reactivity and the chlorinated products. Chlorocarbazoles generated from carbazole were identified by a comprehensive two-dimensional gas chromatography-mass spectrometry method, and their molecular structures were predicted by the Fukui index of electrophilic attack, $f^{-1}(r)$. As a result, the comprehensive chlorination pathway of carbazole was extrapolated with a total of nine chlorocarbazoles identified, including two monochlorocarbazoles (3-chlorocarbazole and 1chlorocarbazole), four dichlorocarbazoles (3,6-dichlorocarbazole, 1,6-dichlorocarbazole, 1,3dichlorocarbazole and 1,8-dichlorocarbazole), two trichlorocarbazoles (1,3,6-trichlorocarbazole and 1,3,8-trichlorocarbazole) and one tetrachlorocarbazole (1,3,6,8-tetrachlorocarbazole). The $f^{-1}(\mathrm{r})$ value indicates that the $\mathrm{C} 1, \mathrm{C} 3, \mathrm{C} 6$ and $\mathrm{C} 8$ atoms of carbazole were the favored positions for electrophilic attack, with the $\mathrm{C} 3$ and $\mathrm{C} 6$ atoms being the predominant sites for chlorine substitution. 3-Chlorocarbazole, 3,6dichlorocarbazole, 1,3,6-trichlorocarbazole and 1,3,6,8-tetrachlorocarbazole were the major analogues of each chlorocarbazole group; however, the production of minor analogues such as 1-chlorocarbazole and 1,6-dichlorocarbazole should not be overlooked. The chlorination of carbazole was a pseudo first order reaction with a reaction rate of $0.1836 \mathrm{nmol} /(\mathrm{L} \cdot \mathrm{h})$ and half-life of $3.77 \mathrm{~h}\left(\mathrm{pH}=6.4, \mathrm{Cl}_{2}=4.7 \mathrm{mg} / \mathrm{L}\right)$, and the chlorination rate of carbazole was approximately 5 times faster than that of an known chlorination precursor pyrene. These results showed that Fukui index was efficacious to predict the chlorination sites of aromatic compounds, and that carbazole is readily transformed into toxic chlorocarbazoles in drinking water treatment plants that use chlorine as a disinfection agent.
\end{abstract}

(๔) 2017 Elsevier Ltd. All rights reserved.

\section{Introduction}

Carbazole is an environmental carcinogenic and mutagenic compound that structurally resembles polycyclic aromatic hydrocarbons (PAHs). Carbazole has been detected in various

\footnotetext{
This paper has been recommended for acceptance by B. Nowack.

* Corresponding author. Key Laboratory of Drinking Water Science and Technology, Research Center for Eco-Environmental Sciences, Chinese Academy of Sciences, Shuangqing Road 18\#, Haidian District, Beijing 100085, China.

E-mail address: dhwang@rcees.ac.cn (D. Wang).
}

environments such as atmospheric particles, soil, sediment and water (Esen et al., 2010; Grosser et al., 1995; Carlsson and. Östman, 1997; Stackelberg et al., 2007; Kochany and Maguire, 1994). Carbazole originates in the environment from anthropological sources such as the petroleum industry and the manufacturing of carbazole-based industrial materials such as dyes, medicines and insecticides (Benedik et al., 1998). Additionally, carbazole may form from $\mathrm{N}$-fertilizers in an analogy to the formation of PCDFs from pesticides due to the structural similarity between carbazole and PCDFs (Altarawneh et al., 2009a). Also, catalytic-assisted coupling of direct precursors such as aniline may produce carbazole 
(Altarawneh et al., 2008). However, the fate of carbazole in the environment is still unclear (Esen et al., 2010; Benedik et al., 1998). Recently, the presence of carbazole's chlorinated derivatives in the environment has drawn increased attention due to their toxicity and wide spread occurrence (Trobs et al., 2011; Reischl et al., 2005; Kronimus et al., 2004; Grigoriadou and Schwarzbauer, 2011; Guo et al., 2014).

As the chlorinated derivatives of carbazole, chlorocarbazoles are considered to be a class of emerging organic contaminates, which are structurally similar to chlorinated dibenzofurans (Riddell et al., 2015). Several chlorocarbazole analogues, including 3chlorocarbazole and 3,6-dichlorocarbazole, have been shown to induce dioxin-like activity using ethoxyresorufin-O-deethylase (EROD) induction in rat hepatoma cells and CYP1A1 and CYP1B1 gene expression in Ah-responsive breast cancer cells (Trobs et al., 2011; Riddell et al., 2015; Mumbo et al., 2015). Although it has been found that halogenated indigo dyes are possible sources of bromocarbazoles in the environment, the origin of chlorocarbazoles is still under debate (Parette et al., 2015), as they may come from both natural and anthropologic sources (Luk et al., 1983). Recently, Altarawneh et al (Altarawneh and Dlugogorski, 2015). found agreement between computational predictions and environmental profiles of chlorocarbazoles and suggested the formation of chlorocarbazoles by electrophilic substitution. To date, the occurrence of chlorocarbazoles has only been reported in soil (Trobs et al., 2011; Reischl et al., 2005) and sediment (Kronimus et al., 2004; Grigoriadou and Schwarzbauer, 2011), and the possible presence of chlorocarbazoles in aqueous environments has not been discussed. Considering the formation mechanism of chlorocarbazoles proposed by Altarawneh et al. (Altarawneh and Dlugogorski, 2015), we believe that the chlorination disinfection of raw water containing carbazole in drinking water treatment plants is a possible source of chlorocarbazoles in drinking water.

Chlorination is a globally used method for the effective removal of hazardous microorganisms in drinking water. Apart from wellknown disinfection by-products such as trihalomethanes (THMs) and haloacetic acids (HAAs), active chlorine residues can also transform toxic micro-organic pollutants in source water into their chlorinated derivatives, which may be more toxic and resistant to being metabolized (Deborde and von Gunten, 2008; Lane et al., 2015; Hu et al., 2006). Previous studies have confirmed that PAHs type compounds can be transformed into chlorinated polycyclic aromatic hydrocarbons (Cl-PAHs) during the chlorination disinfection of drinking water due to the electrophilic substitution reaction between the PAHs and active chlorine species, especially hypochlorous acid (HClO) (Hu et al., 2006; Mori et al., 1991; Georgi et al., 2007; Nakamura et al., 2007). Similar to PAHs, carbazole has an aromatic structure, which could easily participate in the electrophilic substitution reaction. Since the presence of carbazole has been reported in source water (Stackelberg et al., 2007), we believe carbazole is a potential precursor of chlorocarbazoles in drinking water when chlorine is used as a disinfection agent in drinking water treatment plants. To our knowledge, only Lin et al (Lin and Carlson, 1984). in 1984 and Onodera et al. (1989). in 1989 subjected carbazole to aqueous chlorination using sodium hypochlorite and found that carbazole produced chlorocarbazoles with 1-4 chlorine atom substitutions, but both studies were unable to elucidate the specific structures of the chlorocarbazoles formed during aqueous chlorination. Furthermore, the reactivity of carbazole, which determines the formation potential of chlorocarbazoles, has not been studied. This lack of information may result from the complexity of chlorocarbazole isomers and the lack of proper analytical methods and reference materials. However, with the recent increasing attention to chlorocarbazole's toxicity and environmental presence (Riddell et al., 2015; Mumbo et al.,
2015; Parette et al., 2015), it is crucial to characterize the reactivity and chlorinated products of carbazole during aqueous chlorination.

Therefore, in this study, the aqueous chlorination of carbazole was carried out to determine its reactivity with active chlorine and to characterize the chlorocarbazole analogues using a comprehensive two-dimensional gas chromatography-mass spectrometry $(\mathrm{GC} \times \mathrm{GC}-\mathrm{MS})$ method, and the molecular structures of chlorocarbazole analogues were predicted by the Fukui index of electrophilic attack.

\section{Materials and methods}

\subsection{Aqueous chlorination}

For each treatment, sodium hypochlorite solution (8\%-12\% free chlorine) and $1 \mathrm{~L}$ of ultrapure water generated by a Milli-Q system (Millipore, USA) were mixed in amber glass. The free chlorine concentration was measured by a pocket colorimeter for free chlorine (HACH, USA). Sulfuric acid $(0.5 \mathrm{M})$ was used to adjust the solution to the desired $\mathrm{pH}$ level, which was measured by a $\mathrm{pH}$ meter (HACH, USA). Immediately, a mixed solution (200 nmol/L) containing carbazole and pyrene (Accustandard, USA) was added to start the chlorination reaction with an initial concentration of $5 \mathrm{nmol} / \mathrm{L}$ at room temperature $\left(20^{\circ} \mathrm{C}\right)$. Two $\mathrm{pH}$ levels were examined (3.8 and 6.4). For $\mathrm{pH}=6.4$, eight reaction times $(0.5 \mathrm{~h}, 1 \mathrm{~h}, 2 \mathrm{~h}$, $4 \mathrm{~h}, 8 \mathrm{~h}, 12 \mathrm{~h}, 24 \mathrm{~h}$ and $48 \mathrm{~h}$ ) were studied; for $\mathrm{pH}=3.8$, ten reaction times ( $2 \mathrm{~min}, 5 \mathrm{~min}, 10 \mathrm{~min}, 20 \mathrm{~min}, 40 \mathrm{~min}, 80 \mathrm{~min}, 160 \mathrm{~min}$, $320 \mathrm{~min}, 12 \mathrm{~h}$ and $24 \mathrm{~h}$ ) were studied. After each reaction time, excess sodium thiosulfate $\left(\mathrm{Na}_{2} \mathrm{~S}_{2} \mathrm{O}_{4}\right)$ was added to terminate the reaction. Each sample had three replicates. Before solid phase extraction (SPE), all samples were spiked with a surrogate standard (Phenanthrene-d10) to monitor the overall recovery efficiency. To properly enrich the unknown compounds formed after chlorination, a C18 cartridge (500 mg, Supelco, USA) coupled with a HLB cartridge (500 mg, Waters, USA) was used for SPE to ensure the proper extraction of possible polar and non-polar products. After extraction, the cartridges were eluted with $10 \mathrm{~mL}$ of dichloromethane (HPLC grade), and the extracts were evaporated under a gentle stream of nitrogen to $0.5 \mathrm{~mL}$ and then stored at $-20^{\circ} \mathrm{C}$ for further instrumental analysis.

\subsection{Instrumental analysis}

GC $\times$ GC-MS was used for the identification of chlorinated derivatives. The apparatus consisted of an Agilent 7890 gas chromatography and an Agilent 5975C quadrupole MSD. Modulation was accomplished by a loop-type cryogenic modulator (Zoex, USA). The column used for separation was a standard non-polar/polar set. The first column was a DB-5 MS column $(30 \mathrm{~m} \times 0.25 \mathrm{~mm} \times 0.25 \mu \mathrm{m}$, Agilent, USA), and the second column was a BPX-50 column $(2 \mathrm{~m} \times 0.1 \mathrm{~mm} \times 0.1 \mu \mathrm{m}$, SGE, USA). The modulation period was $6 \mathrm{~s}$, and the flow of cooling nitrogen was $5 \mathrm{~L} / \mathrm{min}$. The hot jet was programmed to increase from $150{ }^{\circ} \mathrm{C}$ to $350{ }^{\circ} \mathrm{C}$ at a rate of $3.0^{\circ} \mathrm{C} /$ min and with a hot pulse of $400 \mathrm{~ms}$. The oven temperature was programmed with an initial temperature of $60{ }^{\circ} \mathrm{C}$, then ramped at $3.0^{\circ} \mathrm{C} / \mathrm{min}$ to $300{ }^{\circ} \mathrm{C}$, and held for $10 \mathrm{~min}$. Helium was used as the carrier gas with a $1.0 \mathrm{~mL} / \mathrm{min}$ flow rate in constant flow mode, and $1 \mu \mathrm{L}$ of sample was injected in splitless mode. Quadrupole MSD was used in scan mode over a mass range from $100 \mathrm{amu}$ to $500 \mathrm{amu}$, the mass acquisition rate was $12500 \mathrm{amu} / \mathrm{sec}$, and the scan rate was 19.8 spectra/sec. Data were processed by the GC Image V 2.2 software.

GC-MS was used for the quantification of carbazole and pyrene. The apparatus consisted of an Agilent 6890 gas chromatography 
and an Agilent 5975B quadrupole MSD. The column was a DB-5 MS column $(30 \mathrm{~m} \times 0.25 \mathrm{~mm} \times 0.25 \mu \mathrm{m}$, Agilent, USA). The oven temperature was programmed with an initial temperature of $50^{\circ} \mathrm{C}$, held for $2 \mathrm{~min}$, then ramped at $10^{\circ} \mathrm{C} / \mathrm{min}$ to the final temperature of $300{ }^{\circ} \mathrm{C}$, and held for $5 \mathrm{~min}$. Helium was used as the carrier gas with a $1.0 \mathrm{~mL} / \mathrm{min}$ flow rate in constant flow mode, and $1 \mu \mathrm{L}$ of sample was injected in splitless mode. Quantification was performed in SIM mode. In this study, the recoveries of carbazole and pyrene were $101 \pm 9 \%$ and $102 \pm 6 \%(n=3)$, respectively. The recovery of surrogate Phenanthrene-d10 was $98 \pm 11 \%(n=57)$. The limit of quantifications (LOQs) of carbazole and pyrene were $2 \mathrm{ppb}$ and $0.5 \mathrm{ppb}$, respectively, which were set to be the 10 -fold of signal to noise ratio $(\mathrm{S} / \mathrm{N})$.

\subsection{Computational methodology}

The Fukui index for electrophilic attack (Parr and Yang, 1984), $f^{-1}(r)$, describes the reactivity of a specific site within a molecule. In this study, the $f^{-1}(\mathrm{r})$ value was used to predict the favored chlorine substitution sites in carbazole and its chlorinated derivatives. Gibbs free energy of formation $\left(\Delta_{f} G^{\bigcirc}\right.$ 298.15) was applied here to evaluate the thermodynamic stability of chlorinated carbazole congeners. The Materials Studio program was used to perform the theoretical calculations in this study, $f^{-1}(r)$ and Gibbs free energy of formation were estimated by the Dmol3 module based on the generalized gradient approximation (GGA), PBE functional and DNP 4.4 basis set. All the isomers including parent carbazole were geometrically optimized and were computed in the solvent model (water).

\section{Results and discussion}

\subsection{Reactivity of carbazole}

To study the reactivity of carbazole, experiments were carried out under relatively mild conditions $\left(\mathrm{pH}=6.4, \mathrm{Cl}_{2}=4.7 \mathrm{mg} / \mathrm{L}\right)$ to approximate typical drinking water treatment conditions. Generally, the reaction rate of pyrene and carbazole with active chlorine depends on the aqueous $\mathrm{pH}$ value and the dosage of pyrene, carbazole and chlorine. In this study, the $\mathrm{pH}$ value and free chlorine concentration were $6.4 \pm 0.1(\mathrm{n}=24)$ and $4.7 \pm 0.3 \mathrm{mg} / \mathrm{L}(\mathrm{n}=24)$ before chlorination, respectively, and $6.4 \pm 0.3(\mathrm{n}=24)$ and $4.5 \pm 0.2 \mathrm{mg} / \mathrm{L}(\mathrm{n}=24)$ after chlorination, respectively. Due to small variations in the $\mathrm{pH}$ level and free chlorine concentration, we deemed the reaction conditions to be consistent over the entire chlorination process. Thus, the reaction rates of pyrene and carbazole with active chlorine solely related to their aqueous concentration, which can be described with the following function:

$\frac{d C_{p y}}{d t}=-k_{p y} C_{p y}$

$\frac{d C_{c b z}}{d t}=-k_{c b z} C_{c b z}$

Where $C_{p y}, C_{c b z}, k_{p y}$ and $k_{c b z}$ are the concentrations and reaction rate constants of pyrene and carbazole. The concentration variation of carbazole and pyrene throughout the reaction period can be fitted with the first-order exponential decay model. Fig. 1 shows the curve fittings using ExpDec1 in Origin 13. The adjusted R-square values of pyrene and carbazole were both 0.998 . The reaction rates of carbazole and pyrene were $0.1836 \mathrm{nmol} /(\mathrm{L} \cdot \mathrm{h})$ and $0.0362 \mathrm{nmol} /$ $(\mathrm{L} \cdot \mathrm{h})$, respectively, and their half-lives were $3.77 \mathrm{~h}$ and $19.10 \mathrm{~h}$, respectively. These results showed that under the same reaction conditions, the chlorination of carbazole was approximately five times faster than that of pyrene.

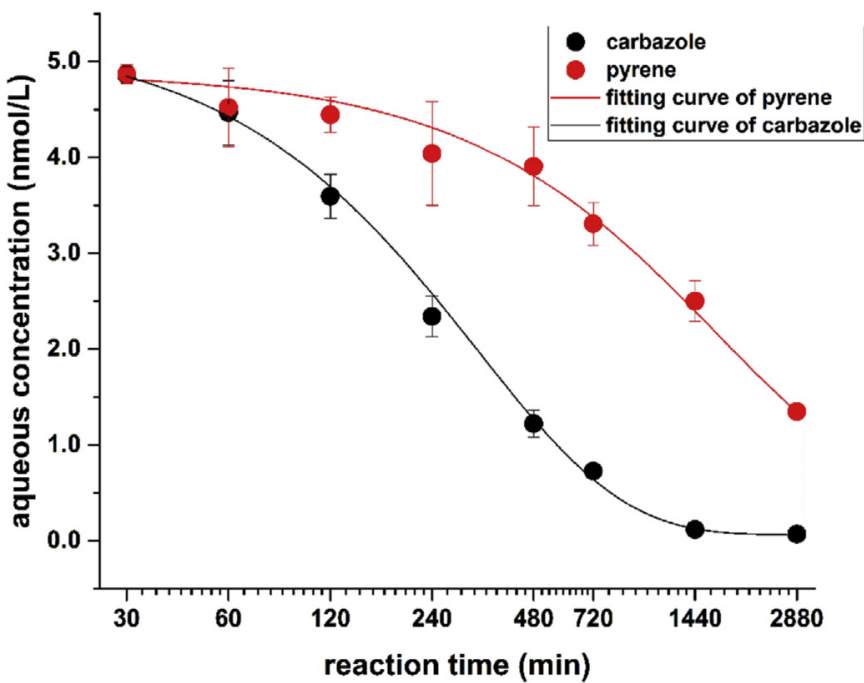

Fig. 1. Fitting curves of the concentrations of carbazole and pyrene during aqueous chlorination.

Under simulated laboratory conditions, many PAHs can be transformed to their corresponding chlorinated derivatives ( $\mathrm{Hu}$ et al., 2006; Nakamura et al., 2007; Onodera et al., 1989; Oyler et al., 1983; Johnsen et al., 1989). However, it has also been reported that each PAHs has a different reactivity during aqueous chlorination (Mori et al., 1991; Nakamura et al., 2007). Mori et al. found that the structure-related reactivity of five tetracyclic aromatic hydrocarbons, including benz[a]anthracene, benzo[c]phenanthrene, chrysene, pyrene and triphenylene, were distinct and depended on their ionization potential (Mori et al., 1991). Alternatively, some of the most common PAHs found in aqueous chlorination, such as fluorene and fluoranthene, were shown to not produce chlorinated fluorene and fluoranthene at all under typical drinking water treatment conditions (Tillner et al., 2013). For carbazole, its aromatic structure makes it a potential precursor for chlorocarbazoles in drinking water. Although Onodera et al. (1989). and Lin et al (Lin and Carlson, 1984). found that carbazole reacts with active chlorine species under laboratory conditions, it is crucial to evaluate the reactivity of carbazole under typical drinking water treatment conditions with an environmental benchmark. Recently, 1-chloropyrene has been detected in drinking water distribution systems as the chlorination product of residue chlorine and pyrene (Tillner et al., 2013). The chlorination mechanism of pyrene, which consists of chlorine atom substitution reactions on the benzene ring through electrophilic attack by active chlorine, is the same as that of carbazole. Therefore, it is suitable to compare the chlorination reactivity of pyrene and carbazole to explore the possible occurrence of chlorocarbazole in drinking water. Our results showed that carbazole possesses a higher reactivity than pyrene under typical drinking water treatment conditions and indicated the possible presence of chlorocarbazole in chlorinated drinking water when carbazole was present in the source water.

\subsection{Characterization of chlorinated carbazoles by GC $\times$ GC-MS and the Fukui index}

The identification of chlorinated derivatives was performed under relatively radical experimental conditions $(\mathrm{pH}=3.8$, $\mathrm{Cl}_{2}=12 \mathrm{mg} / \mathrm{L}$ ) because higher dosages of active chlorine and low $\mathrm{pH}$ levels accelerate the chlorination reaction without altering the transformation products (Nakamura et al., 2007), and the chlorination reaction proceeded more readily, which is suitable for the 
comprehensive identification of products at low pH levels. In this study, two monochlorocarbazoles (cl-cbz), four dichlorocarbazoles (dicl-cbz), two trichlorocarbazoles (tricl-cbz) and one tetrachlorocarbazole (tetracl-cbz) were identified as the transformation products of carbazole according to the mass spectra from the twodimensional chromatograms presented in Fig. 2.

For monocyclic aromatic hydrocarbons compounds, the chlorination positions are relatively easy to predict (Deborde and von Gunten, 2008; Hu et al., 2002). For example, the chlorination of phenols were well documented and the chlorination reaction of phenols mainly occurs in ortho or para position due to the activating ortho/para directing hydroxyl group (Gallard and von Gunten, 2002; Acero et al., 2005). However, chlorination on polycyclic aromatic hydrocarbons compounds are complicated due to the multiple possible reaction sites. Similar to PAHs, the aromatic structure of carbazole allows for electrophilic attack. The chlorination reaction between carbazole and active chlorine species, in this case, hypochlorite acid ( $\mathrm{HClO})$, is an electrophilic substitution reaction since the chlorine atom in $\mathrm{HClO}$ is in the electron deficient state (Deborde and von Gunten, 2008). Carbazole contains eight possible carbon atom sites for chlorine substitution, shown in Fig. 3, and the favored site for substitution was determined by the reactivity of each possible site. The Fukui index for electrophilic attack (Parr and Yang, 1984), $f^{-1}(\mathrm{r})$, which describes the reactivity of a specific site within a molecule, provides an approach to explore the intrinsic reactivity within a compound (Ghomri and Mekelleche, $2010)$, and in this case, the $f^{-1}(r)$ value was used to predict the favored chlorine substitution site in carbazole and its chlorinated derivatives.

First, theoretical calculations were performed to generate $f^{-1}(r)$ values for each possible substitution site in carbazole, and the major chlorocarbazole analogues were predicted based on the magnitude of the $f^{-1}(\mathrm{r})$ value and experimental data. Considering the number of chlorocarbazoles detected in this study, we proposed the chlorination pathway shown in Fig. 3. Higher $f^{-1}(r)$ values indicate the most probable sites for chlorine substitution and lead to higher production quantities. According to the $f^{-1}(r)$ value of each possible substitution site in Fig. 3, the favored site for chlorination through electrophilic substitution follows the order of $\mathrm{C} 3>\mathrm{C} 1>\mathrm{C} 4>\mathrm{C} 2$. For the two cl-cbz species identified here, we believe the two monochloro analogues were 3 -chlorocarbazole and 1-chlorocarbazole, with 3-chlorocarbazole being the predominant specie. 3-Chlorocarbazole and 1-chlorocarbazole were then further chlorinated into four different dicl-cbz species. According to the $f^{-1}(r)$ of 3-chlorocarbazole and 1-chlorocarbazole, C1, C3, C6 and C8 were the favored positions for chlorine substitution, with C3 and C6 being the major substitution sites. Thus, dichlorocarbazoles formed from 3-chlorocarbazole in the following order: 3,6dichlorocarbazole> 1,6-dichlorocarbazole> 1,3-dichlorocarbazole. Dichlorocarbazoles formed from 1-chlorocarbazole in the following order: 1,6-dichlorocarbazole> 1,3-dichlorocarbazole> 1,8dichlorocarbazole. Thus, we obtained four different dicl-cbz species, which corresponds with the number of dicl-cbz species identified in the GC $\times$ GC chromatogram, and their production follows the order of 3,6-dichlorocarbazole $>1,6$-dichlorocarbazole $>$ 1,3-dichlorocarbazole> 1,8-dichlorocarbazole. For the abovementioned dicl-cbz species, the favored sites for chlorine substitution are still $\mathrm{C} 1, \mathrm{C} 3, \mathrm{C} 6$ and $\mathrm{C} 8$, and further chlorination of dicl-cbz at these sites will lead to two different tricl-cbz species $(1,3,6-$ trichlorocarbazole and 1,3,8-trichlorocarbazole). 1,3,6-
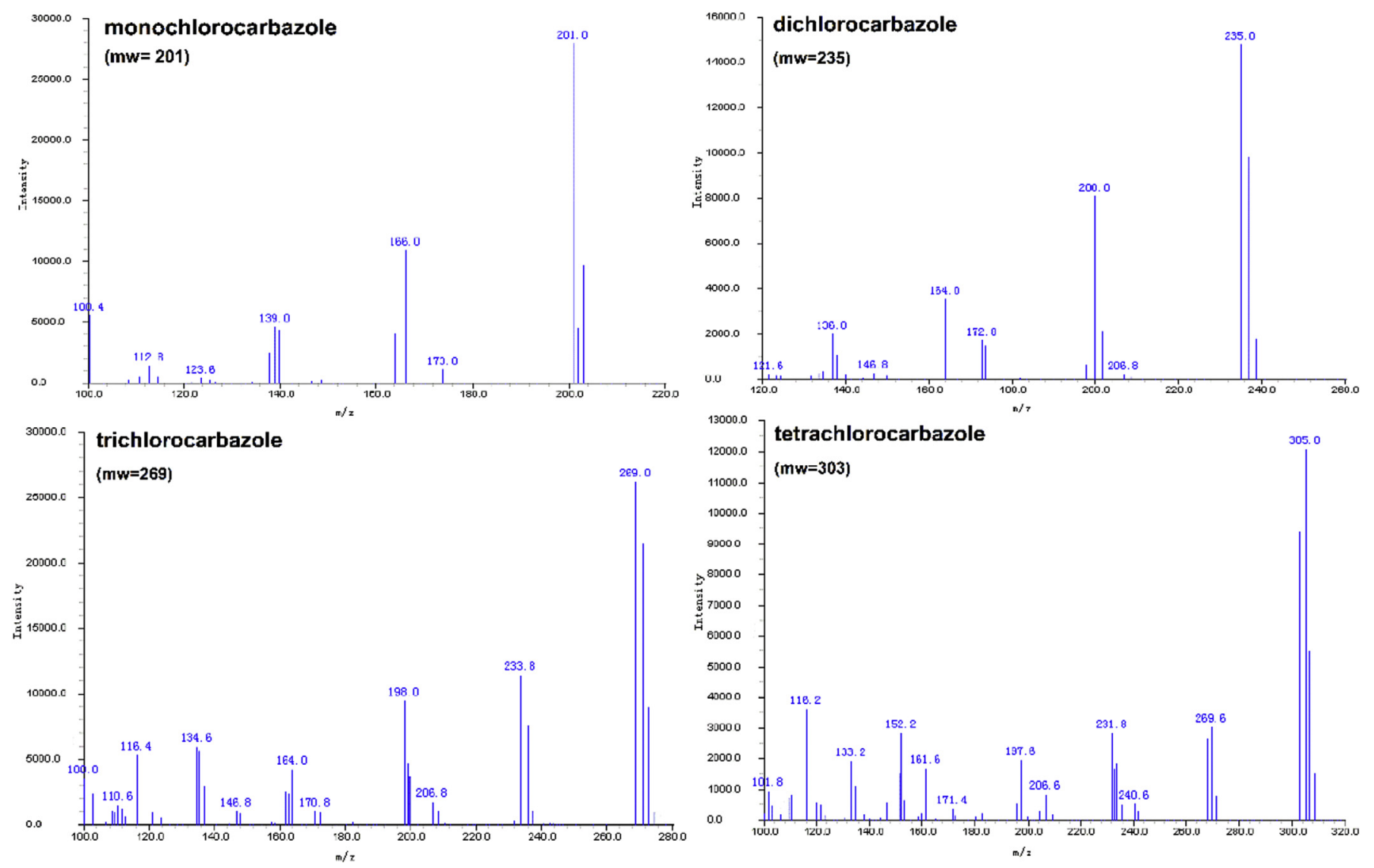

Fig. 2. Mass spectrums of monochlorocarbazole, dichlorocarbazole, trichlorocarbazole and tetrachlorocarbazole. 


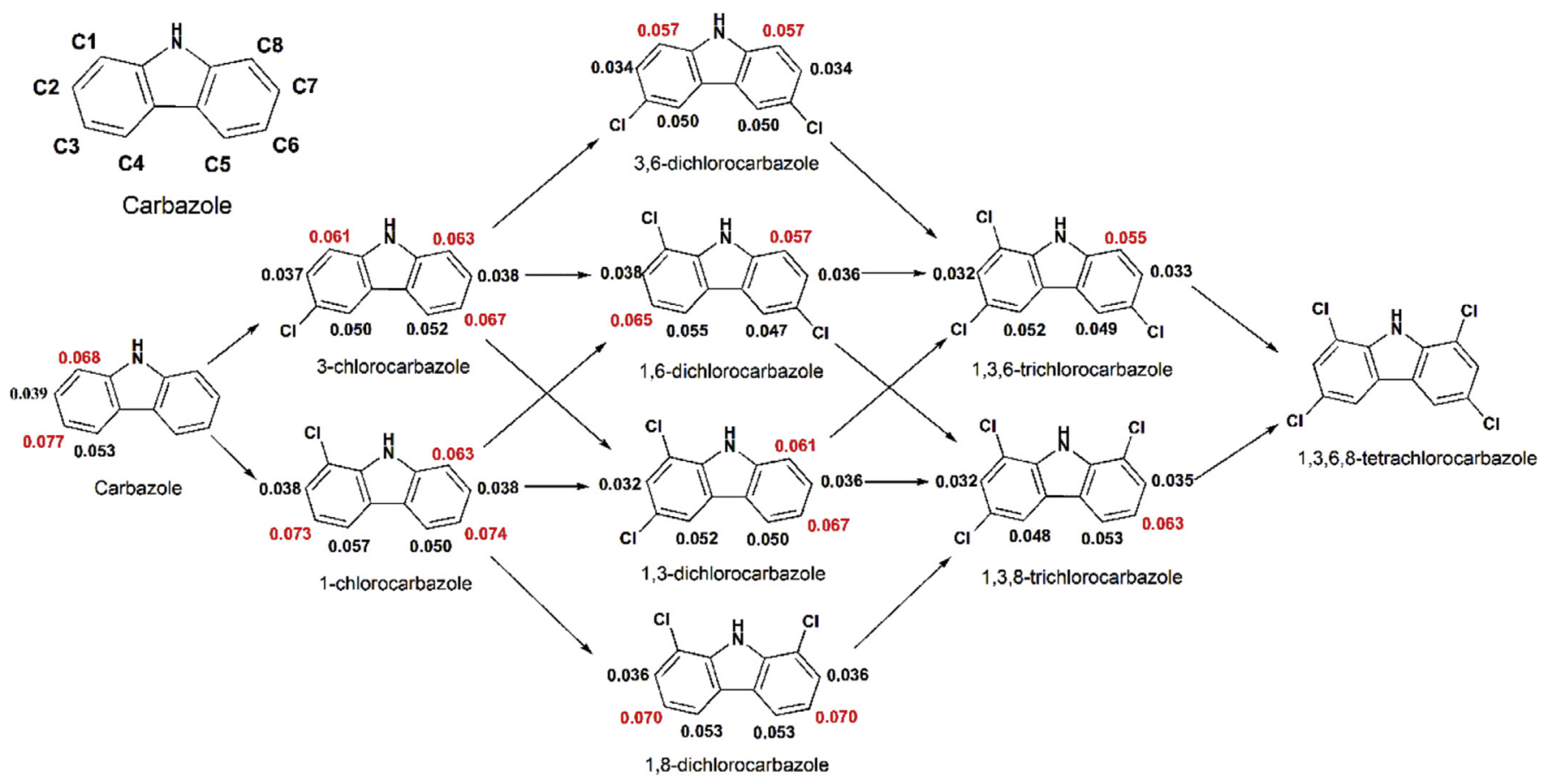

Fig. 3. Proposed chlorination pathway of carbazole during aqueous chlorination.

Trichlorocarbazole was the major product due to the dominant production of 3,6-dichlorocarbazole and the higher $f^{-1}(r)$ value of the C3 and C6 sites. Finally, these two tricl-cbz species will be further chlorinated to produce the same 1,3,6,8tetrachlorocarbazole molecule.

Next, the peak areas of the chlorocarbazoles and the theoretical results discussed above were combined to identify the chlorocarbazole peaks detected in the two-dimensional chromatogram. Fig. 4 shows the simplified two-dimensional chromatogram of chlorocarbazoles. It can be seen that the chlorocarbazole species are well separated in the structural chromatogram. The retention time of each chlorocarbazole category (cl-cbz, dicl-cbz and tricl$\mathrm{cbz}$ ) in the first dimension were distinctively different. Since

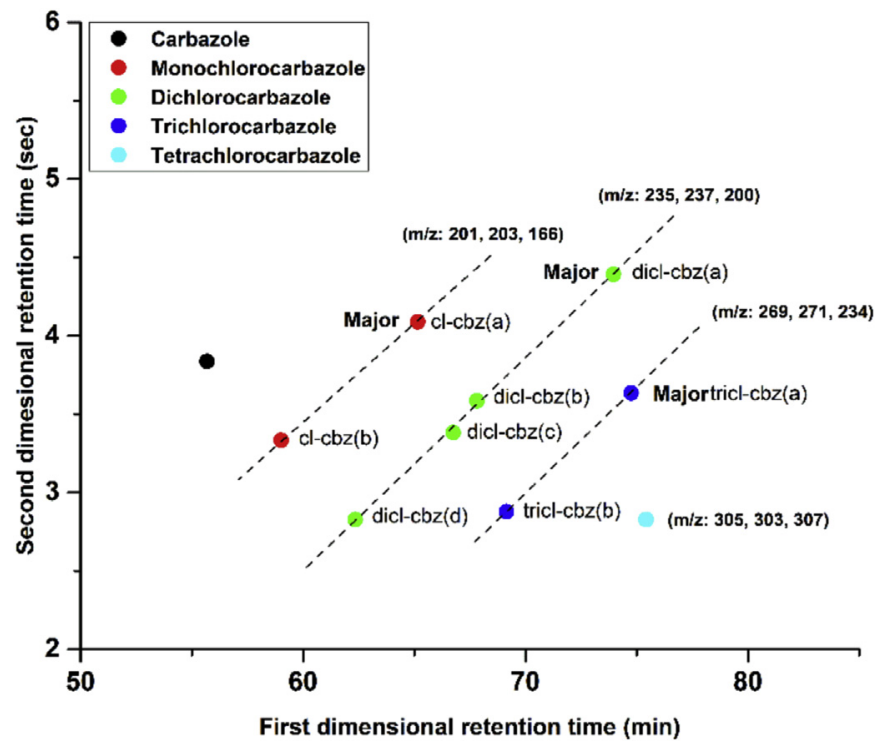

Fig. 4. Simplified two-dimensional chromatogram of chlorocarbazole analogues. separation in comprehensive two-dimensional gas chromatography is mainly based on volatility and polarity, we believe that the different chlorine substitution sites greatly influence the polarity and volatility of the resultant chlorocarbazole. Due to the lack of standard references for chlorocarbazoles, we identified each chlorocarbazole analogue based on the peak area generated from the mass spectrum in select ion mode (SIM) and the predicted structure from $f^{-1}(\mathrm{r})$ discussed above. Fig. 5 shows the peak area of monochlorocarbazoles, dichlorocarbazoles and trichlorocarbazoles. For the two detected monochlorocarbazoles, we believe the major product cl-cbz(a) was 3-chlorocarbazole and cl-cbz(b) was 1chlorocarbazole. Although 3-chlorocarbazole was the dominant species, the production of 1-chlorocarbazole cannot be overlooked. After 2 min of reaction, the product ratio between these two analogues was approximately $4: 1$, meaning that approximately $20 \%$ of the carbazole was transformed to 1-chlorocarbazole. According to the prediction using the $f^{-1}(\mathrm{r})$ values and the peak areas of the four dichlorocarbazoles from Fig. 5, dicl-cbz(a) and dicl-cbz(b) were 3,6dichlorocarbazole and 1,6-dichlorocarbazole, respectively, with an approximate ratio of 7:3 after 20 min of reaction; however, the production of both $\operatorname{dicl}-\mathrm{cbz}(\mathrm{c})$ and $\operatorname{dicl}-\mathrm{cbz}(\mathrm{d})$ were too low to be distinguished. However, according to the distribution pattern of carbazoles in Fig. 4, it seems that when a chlorine atom was attached at the $\mathrm{C} 1$ site, the retention time of chlorocarbazole was shorter than that with chlorine substituted at the $\mathrm{C} 3$ site; thus, we believe that dicl-cbz(d) was 1,8-dichlorocarbazole and, consequently, $\operatorname{dicl}-\operatorname{cbz}(\mathrm{c})$ was 1,3-dichlorocarbazole. For the trichlorocarbazoles, tricl-cbz(a) and tricl-cbz(b) are identified as 1,3,6trichlorocarbazole and 1,3,8-trichlorocarbazole, respectively, with an approximate ratio of 9:1 after $160 \mathrm{~min}$ of reaction. Among all the chlorocarbazole analogues, 3,6-dichlorocarbazole was confirmed by comparison to a reference material and proved to be the dominant dichlorocarbazole species, which means that 3chlorocarbazole was the major monochlorocarbazole species and that C3 and C6 were indeed the most favored sites for chlorine substitution. 


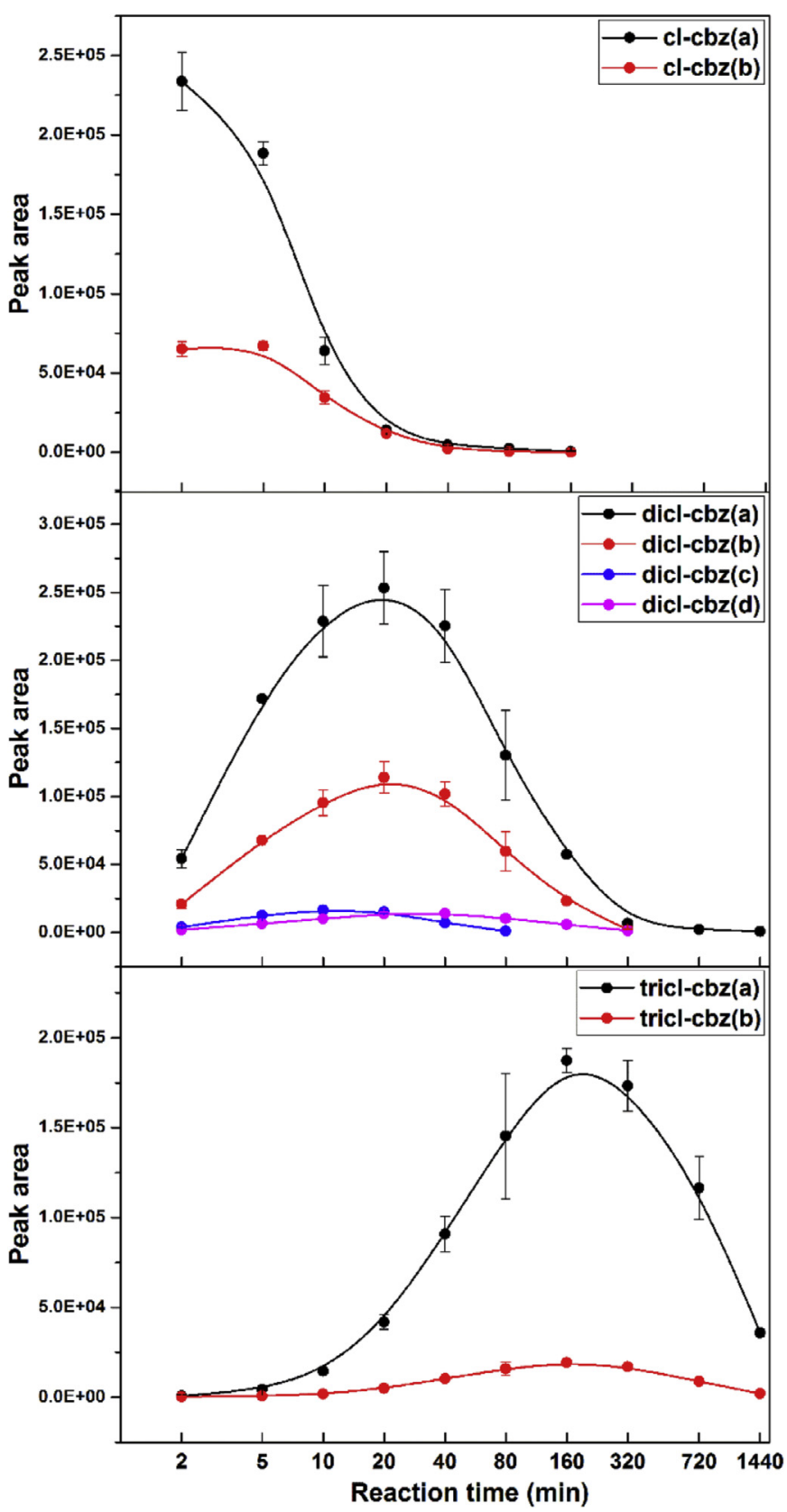

Fig. 5. Peak areas of monochlorocarbazoles, dichlorocarbazoles and trichlorocarbazoles.

Although $f^{-1}(\mathrm{r})$ was proved to be a useful molecular descriptor for the chlorination of carbazole in this research, previous studies pointed out that the chlorination pattern could also be under thermodynamic control. Congeners with higher thermodynamic stability are more abundant than least stable isomers (Altarawneh et al., 2009b). In this study, Gibbs free energy of formation was calculated to evaluate the thermodynamic stability of parent and chlorinated carbazole, Table 1 . In each chlorinated carbazole group, the congeners distribution seems to be kinetically driven rather than thermodynamic stability controlled. For instance, the free energy of 3-chlorocarbazole and 1,3,6-trichlorocarbazole, which were determined as major mono- and tri-chlorinated congeners, were larger than 1-chlorocarbazole and 1,3,8-trichlorocarbazole, respectively, which indicates they are thermodynamically less
Table 1

Gibbs free energy of formation of parent and chlorinated carbazoles.

\begin{tabular}{ll}
\hline Compound & $\Delta_{f} G^{\circ} 298.15(\mathrm{kcal} / \mathrm{mol})$ \\
\hline Carbazole & 87.38 \\
3-chlorocarbazole & 79.51 \\
1-chlorocarbazole & 79.414 \\
3,6-dichlorocarbazole & 71.287 \\
1,6-dichlorocarbazole & 73.248 \\
1,3-dichlorocarbazole & 71.34 \\
1,8-dichlorocarbazole & 72.321 \\
1,3,6-trichlorocarbazole & 65.784 \\
1,3,8-trichlorocarbazole & 64.962 \\
1,3,6,8-tetrachlorocarbazole & 57.003 \\
\hline
\end{tabular}

stable than 1-chlorocarbazole and 1,3,8-trichlorocarbazole. Although 3,6-dichlorocarbazole possesses the lowest free energy among four di-chlorinated carbazoles, their thermodynamic stability order is different from the production amount order from this study. The chlorination of carbazole from one to four chlorine substitution is largely kinetically driven, since the distribution of each chlorinated carbazole group variated as a function of reaction time, Fig. 5. However as the chlorine atom substitution increases, the free energy of formation deceases. This suggested that carbazole is able to be transformed into higher chlorinated carbazoles during aqueous chlorination under both chemical kinetics and thermodynamic control.

Our results confirmed with the number of chlorocarbazole formed during aqueous chlorination reported by Lin et al. (Lin and Carlson, 1984). Although Altarawneh et al (Altarawneh and Dlugogorski, 2015). used $f^{-1}(r)$ to generally match the dominant chlorocarbazoles detected in environmental media originating from various sources, to the best of our knowledge, this study is the first to describe the aqueous chlorination products of carbazole based on both experimental results and computational predictions. In addition, according to our study, 3-chlorocarbazole, 3,6dichlorocarbazole, 1,3,6-trichlorocarbazole and 1,3,6,8tetrachlorocarbazole were found as the major products, but the production of minor products such as 1-chlorocarbazole, 1,6dichlorocarbazole and 1,3,8-trichlorocarbazole cannot be overlooked. It should be noted that up to four chlorine atoms were substituted on carbazole under radical conditions, however, under typical drinking water treatment conditions, monochlorocarbazoles, including 3-chlorocarbazole and 1-chlorocarbazole, are the most probable chlorocarbazole species in drinking water.

\section{Conclusions}

Collectively, the comparison of the chlorination reaction rate and the half-lives between carbazole and an environmental benchmark pyrene have shown that carbazole possessed higher reactivity over pyrene, and indicates the probable presence of toxic chlorocarbazoles in drinking water. The ability of Fukui index of electrophilic attack to predict chlorine substitution sites was confirmed in the case of carbazole, and for the first time, the comprehensive chlorination pathway of carbazole was elucidated with the combined application of GC $\times$ GC-MS and Fukui index. We believe this application could be further used to identify chlorinated by-products of other possible precursors with aromatic structure during chlorination process. Due to the high reactivity of carbazole during aqueous chlorination and the dioxin-like toxicity of chlorocarbazoles, the presence of chlorocarbazoles in drinking water and their potential hazardous effects to human health deserve more research. 


\section{Acknowledgments}

This study was supported by the National Natural Science Foundation of China (Grant No. 21677155, 51290283).

\section{References}

Acero, J.L., Piriou, P., Von Gunten, U., 2005. Kinetics and mechanisms of formation of bromophenols during drinking water chlorination: assessment of taste and odor development. Water Res. 39 (13), 2979-2993.

Altarawneh, M., Dlugogorski, B.Z., 2015. Formation and chlorination of carbazole, phenoxazine, and phenazine. Environ. Sci. Technol. 49 (4), 2215-2221.

Altarawneh, M., et al., 2008. Adsorption of chlorophenol on the Cu (111) surface: a first-principles density functional theory study. Appl. Surf. Sci. 254 (14), 4218-4224.

Altarawneh, M., et al., 2009a. Pyrolysis of permethrin and formation of precursors of polychlorinated dibenzo-p-dioxins and dibenzofurans (PCDD/F) under nonoxidative conditions. Chemosphere 74 (11), 1435-1443.

Altarawneh, M., et al., 2009b. Mechanisms for formation, chlorination, dechlorination and destruction of polychlorinated dibenzo-p-dioxins and dibenzofurans (PCDD/Fs). Prog. Energy Combust. Sci. 35 (3), 245-274.

Benedik, M.J., et al., 1998. Microbial denitrogenation of fossil fuels. Trends Biotechnol. 16 (9), 390-395.

Carlsson, H., Östman, C., 1997. Clean-up and analysis of carbazole and acridine type polycyclic aromatic nitrogen heterocyclics in complex sample matrices. J. Chromatogr. A 790 (1), 73-82.

Deborde, M., von Gunten, U., 2008. Reactions of chlorine with inorganic and organic compounds during water treatment-Kinetics and mechanisms: a critical review. Water Res, 42 (1-2), 13-51.

Esen, F., Tasdemir, Y., Cindoruk, S.S., 2010. Dry deposition, concentration and gas/ particle partitioning of atmospheric carbazole. Atmos. Res. 95 (4), 379-385.

Gallard, H., von Gunten, U., 2002. Chlorination of phenols: kinetics and formation of chloroform. Environ. Sci. Technol. 36 (5), 884-890.

Georgi, A., et al., 2007. Influence of sorption to dissolved humic substances on transformation reactions of hydrophobic organic compounds in water. I. Chlorination of PAHs. Environ. Sci. Technol. 41 (20), 7003-7009.

Ghomri, A., Mekelleche, S.M., 2010. Reactivity and regioselectivity of fivemembered heterocycles in electrophilic aromatic substitution: a theoretical investigation. J. Mol. Struct. THEOCHEM 941 (1-3), 36-40.

Grigoriadou, A., Schwarzbauer, J., 2011. Non-target screening of organic contaminants in sediments from the industrial coastal area of Kavala City (NE Greece). Water, Air, \& Soil Pollut. 214 (1-4), 623-643.

Grosser, R.J., Vestal, J.R., Warshawsky, D., 1995. Mineralization of polycyclic and Nheterocyclic aromatic compounds in hydrocarbon-contaminated soils. Environ. Toxicol. Chem. 14 (3), 375-382.

Guo, J., et al., 2014. Polyhalogenated carbazoles in sediments of Lake Michigan: a new discovery. Environ. Sci. Technol. 48 (21), 12807-12815.

Hu, J.-y., Aizawa, T., Ookubo, S., 2002. Products of aqueous chlorination of bisphenol
A and their estrogenic activity. Environ. Sci. Technol. 36 (9), 1980-1987.

Hu, J.Y., et al., 2006. Transformation of pyrene in aqueous chlorination in the presence and absence of bromide ion: kinetics, products, and their aryl hydrocarbon receptor-mediated activities. Environ. Sci. Technol. 40 (2), 487-493.

Johnsen, S., Gribbestad, I., Johansen, S., 1989. Formation of chlorinated PAHpossible health hazard from water chlorination. Sci. Total Environ. 81, 231-238.

Kochany, J., Maguire, R., 1994. Abiotic transformations of polynuclear aromatic hydrocarbons and polynuclear aromatic nitrogen heterocycles in aquatic environments. Sci. total Environ. 144 (1-3), 17-31.

Kronimus, A., et al., 2004. Anthropogenic organic contaminants in sediments of the Lippe river, Germany. Water Res. 38 (16), 3473-3484.

Lane, R.F., et al., 2015. Chlorination and chloramination of bisphenol A, bisphenol F and bisphenol A diglycidyl ether in drinking water. Water Res. 79, 68-78.

Lin, S., Carlson, R.M., 1984. Susceptibility of environmentally important heterocycles to chemical disinfection: reactions with aqueous chlorine, chlorine dioxide, and chloramine. Environ. Sci. Technol. 18 (10), 743-748.

Luk, K.-C., et al., 1983. Isolation and identification of" diazepam-like" compounds from bovine urine. J. Nat. Prod. 46 (6), 852-861.

Mori, Y., et al., 1991. Aqueous chlorination of tetracyclic aromatic hydrocarbons: reactivity and product distribution. Chemosphere 22 (5), 495-501.

Mumbo, J., et al., 2015. Persistence and dioxin-like toxicity of carbazole and chlorocarbazoles in soil. Environ. Sci. Pollut. Res. Int. 22 (2), 1344-1356.

Nakamura, H., et al., 2007. Reaction of polycyclic aromatic hydrocarbons adsorbed on silica in aqueous chlorine. Environ. Sci. Technol. 41 (7), 2190-2195.

Onodera, S., et al., 1989. Chemical changes of organic compounds in chlorinated water: XVI. Gas chromatographic-mass spectrometric studies of reactions of tricyclic aromatic hydrocarbons with hypochlorite in dilute aqueous solution. J. Chromatogr. A 466, 233-249.

Oyler, A.R., et al., 1983. Chlorine disinfection chemistry of aromatic compounds. Polynuclear aromatic hydrocarbons: rates, products, and mechanisms. Environ. Sci. Technol. 17 (6), 334-342.

Parette, R., et al., 2015. Halogenated indigo dyes: a likely source of 1, 3, 6, 8tetrabromocarbazole and some other halogenated carbazoles in the environment. Chemosphere 127, 18-26.

Parr, R.G., Yang, W., 1984. Density functional approach to the frontier-electron theory of chemical reactivity. J. Am. Chem. Soc. 106 (14), 4049-4050.

Reischl, A., Joneck, M., Dumler-Gradl, R., 2005. Chlorcarbazole in Böden. Umweltwissenschaften Schadst. 17 (4), 197-200.

Riddell, N., et al., 2015. Characterization and Biological potency of mono- to Tetrahalogenated carbazoles. Environ. Sci. Technol. 49 (17), 10658-10666.

Stackelberg. P.E., et al., 2007. Efficiency of conventional drinking-water-treatmen processes in removal of pharmaceuticals and other organic compounds. Sci. Total Environ. 377 (2), 255-272.

Tillner, J., et al., 2013. Simultaneous determination of polycyclic aromatic hydrocarbons and their chlorination by-products in drinking water and the coatings of water pipes by automated solid-phase microextraction followed by gas chromatography-mass spectrometry. J. Chromatogr. A 1315, 36-46.

Trobs, L., et al., 2011. Degradative fate of 3-chlorocarbazole and 3,6-dichlorocarbazole in soil. Environ. Sci. Pollut. Res. 18 (4), 547-555. 